\title{
Factores que incrementan el riesgo de que los jóvenes se vean involucrados en situaciones violentas \\ o delictivas en dos alcaldías de la Ciudad de México
}

Factors that increase the risk

of young people being

involved in violent

or criminal situations

in two city halls of Mexico City

Alejandra Pérez Pérez**

Recibido: 13 de marzo de 2019

Revisado: 22 de mayo de 2019

Aprobado: 10 de junio de 2019

* Cómo citar este artículo: Pérez, A. (2019). Factores que incrementan el riesgo de que los jóvenes se vean involucrados en situaciones violentas o delictivas en dos alcaldías de la Ciudad de México. Revista CIFE: Lecturas de Economía Social, 21(34), 93-114

* Magíster en demografía por El Colegio de México. Socióloga y demógrafa especialista en seguridad y ciudadanía.

Correo electrónico: alojandra@gmail.com.

Orcid: https://orcid.org/0000-0002-9646-6715 


\section{Resumen}

Se presentan dos modelos de regresión logística para explicar el involucramiento de jóvenes en situaciones de riesgo de iniciar una carrera delictiva o tener conductas violentas. Se incluyen habitantes de las ciudades más violentas de México y dos alcaldías de la Ciudad de México para identificar las desventajas que ayuden a explicar su involucramiento en situaciones como el consumo de alcohol y drogas, formar parte de pandillas o poseer armas. Se consideran características en el nivel individual (sexo, edad y ocupación); del hogar (estrato socioeconómico, tipo de hogar, índices de convivencia); y del entorno (percepción de riesgo en la colonia). En general, a nivel nacional y local las desventajas tienen un impacto similar en los jóvenes, siendo el sexo y la edad las que tuvieron el mayor impacto; en la Ciudad de México, características como tipo de hogar, estrato socioeconómico y ocupación no tienen significancia estadística.

Palabras clave: jóvenes, conductas violentas, desventajas acumuladas, criminalidad.

Clasificación JEL: J13, J12, Z13.

\section{Abstract}

Two logistic regression models are presented to explain the involvement of young people in situations of risk of beginning a criminal career or having violent behavior. Inhabitants of the most violent cities in México and two city halls in Mexico City are included. The aim is to identify disadvantages that help explain their involvement in situations as alcohol and drug use, being part of gangs or possessing weapons. Characteristics at individual level (sex, age and occupation); household level (socioeconomic stratum, type of household, cohabitation rates); and the environment (perception of risk in the neighborhood) are considered. In general, at the national and local levels disadvantages have a similar impact on young people, with sex and age having the greatest impact. In Mexico City, characteristics like type of home, socioeconomic stratum and occupation have no statistical significance.

Keywords: Violent behavior, youth, accumulated disadvantages, criminality.

Classification JEL: J13, J12, Z13. 


\section{Introducción}

La delincuencia y criminalidad son características persistentes en México a nivel nacional. Los delitos con mayor reporte año con año son el robo en la vía pública y el transporte público, la extorsión, el fraude, el robo total o parcial de vehículos, las amenazas verbales, el robo a casa habitación y las lesiones; aunado a ello, la inseguridad es percibida como el principal problema a nivel estatal por el $64.5 \%$ de la población mexicana (ENVIPE, 2018).

De acuerdo con cifras del Secretariado Ejecutivo (2019), tan solo en la Ciudad de México (CDMX), en el 2018 se tuvo denuncias de 234677 delitos del fuero común. Cifra que incrementó $26.7 \%$ desde 2011 y 15 \% desde 2017. Esta tendencia supera la observada a nivel nacional; mientras la tasa de víctimas de delito por cada 100000 habitantes a nivel nacional aumentó $5.5 \%$ entre 2015 y 2018 (pasando de 28202 a 38475), la tasa en la capital del país aumentó 11.9 \% (de 38475 a 43 069) (ENVIPE, 2018).

Ante este contexto de violencia, el tema ha sido abordado cada vez con mayor intensidad desde distintos ámbitos de la academia, tanto para describir su comportamiento como para identificar y analizar las relaciones causales asociadas.

Este trabajo se ubica en la segunda tendencia. Se avoca a identificar factores asociados a las conductas violentas en un contexto donde la desigualdad y la exclusión polarizan cada vez más las ciudades, las cuales dan pie al involucramiento de sus habitantes en actividades ilícitas y, a su vez, derivan en la reducción de la cohesión social (Nava, 2014).

Entre los distintos esfuerzos que se han realizado por el gobierno mexicano para responder al clima de inseguridad actual se encuentra la Encuesta de cohesión social para la prevención de la violencia y la delincuencia (Ecopred) (INEGI, 2014). Solo se realizó un levantamiento de esta encuesta en el 2014, enfocado en las ciudades con mayores niveles de violencia y delincuencia. Una de las principales cualidades de la Ecopred es que considera situaciones en distintos niveles de la vida de los jóvenes entrevistados para identificar factores de riesgo comúnmente asociados con el inicio de una carrera delictiva.

Para el análisis de la información se trabaja con el supuesto de que un ambiente violento propicia que, de manera individual, se recurra a conductas violentas en la vida cotidiana para resolver conflictos, situación que incrementa proporcionalmente con el nivel de violencia que haya en el contexto social local. Prueba de ello es que se ha demostrado que el incremento de los homicidios y la reducción de capacidades de los sistemas de justicia suelen estar presentes en sociedades con antecedentes recientes de conflictos civiles (Morrison y Shifter, 2005). 
Particularmente se trabaja con las dos alcaldías ${ }^{1}$ de la Ciudad de México que fueron seleccionadas para ser representadas en la encuesta: Iztapalapa y Gustavo A. Madero. En ellas se ha concentrado el mayor número de habitantes, así como el mayor número de delitos graves en los últimos años. La cantidad de delitos registrados en averiguaciones previas iniciadas o carpetas de investigación se representa en la tabla 1 .

Tabla 1. Cantidad de delitos seleccionados ${ }^{2}$ reportados por alcaldía según total, 2011-2018

\begin{tabular}{lccccc|}
\hline Alcaldía / Año & $\mathbf{2 0 1 5}$ & $\mathbf{2 0 1 6}$ & $\mathbf{2 0 1 7}$ & $\mathbf{2 0 1 8}$ & Total general \\
\hline Iztapalapa & 2843 & 2633 & 2460 & 2818 & 11034 \\
\hline Gustavo A. Madero & 2140 & 2024 & 2103 & 2480 & 8930 \\
\hline Cuauhtémoc & 2085 & 2112 & 2101 & 2183 & 8666 \\
\hline Venustiano Carranza & 1162 & 1192 & 1246 & 1215 & 4890 \\
\hline Álvaro Obregón & 1057 & 945 & 1116 & 1238 & 4518 \\
\hline Tlalpan & 1040 & 1069 & 990 & 1198 & 4382 \\
\hline Benito Juárez & 844 & 921 & 1078 & 939 & 3859 \\
\hline Miguel Hidalgo & 901 & 927 & 999 & 887 & 3815 \\
\hline Coyoacán & 830 & 855 & 892 & 1123 & 3799 \\
\hline Azcapotzalco & 694 & 642 & 691 & 746 & 2835 \\
\hline Iztacalco & 687 & 634 & 597 & 695 & 2673 \\
\hline Xochimilco & 592 & 605 & 556 & 634 & 2436 \\
\hline Tláhuac & 499 & 521 & 545 & 658 & 2286 \\
\hline Cuajimalpa de Morelos & 283 & 232 & 258 & 286 & 1082 \\
\hline La Magdalena Contreras & 235 & 226 & 274 & 295 & 1054 \\
\hline Milpa Alta & 189 & 175 & 224 & 253 & 855 \\
\hline No especificado & 93 & 104 & 67 & 87 & 352 \\
\hline Total general & 16174 & 15817 & 16197 & 17735 & 67466 \\
\hline
\end{tabular}

Fuente. Elaboración propia con datos del Secretariado Ejecutivo del Sistema Nacional de Seguridad Pública (2019)

En la tabla anterior puede corroborarse que la entidad, desde el 2015, ha tenido un incremento constante en la cantidad de delitos registrados, con excepción del 2016, cuando disminuyó. En cuanto al comportamiento por alcaldía, con excepción de cuatro, todas presentan su mayor número de delitos en el 2018. Iztapalapa tuvo la mayor incidencia en el 2015, pero esta parece repuntar en el 2018; por otra parte, Gustavo A.

1 Cuando se realizó la encuesta la demarcación territorial de la capital mexicana se dividía en delegaciones; sin embargo, a partir del 17 de septiembre de 2018 se divide en alcaldías, lo cual implica cambios administrativos, pero mantiene la división geográfica anterior.

2 Se presentan únicamente tres categorías de delitos: los que afectan la libertad y la integridad sexual (abuso sexual, acoso sexual, violación), la vida y la integridad corporal (aborto, homicidio, feminicidio y lesiones), así como la libertad personal (secuestro, tráfico de menores y otros). 
Madero tuvo una ligera reducción en 2016, sin embargo, también ha repuntado en los años subsecuentes.

Para entender los niveles altos de incidencia se plantea un modelo de regresión logística para encontrar variables correlacionadas con el hecho de que los jóvenes hayan tenido conductas que impliquen mayor riesgo de cometer actos delictivos. El objetivo es determinar características en el nivel individual, del hogar y del entorno que ayuden a explicar el hecho de que los jóvenes hayan tenido conductas que conlleven riesgo de cometer actos criminales o delitos en las ya mencionadas alcaldías de la Ciudad de México.

Se espera encontrar que, características como el género, tipo de hogar (nuclear, monoparental o ampliado), el estrato socioeconómico, la forma de relacionarse dentro del hogar y el nivel de peligrosidad del entorno inmediato fuera del hogar (la colonia), inciden en la probabilidad de que los jóvenes hayan tenido conductas riesgosas.

Para entender lo anterior se emplea la perspectiva de desventajas acumuladas (Saraví, 2007), la cual permite reconocer que, a pesar de que exista una relación estadísticamente significativa entre distintas variables, esta no será determinante en el comportamiento de los jóvenes, y que la existencia de varias características de riesgo incrementa la probabilidad, por lo que no solo debe observarse lo que suceda en la vida individual de los jóvenes, sino en todos los ámbitos. Además, se emplea el concepto de socialización primaria por medio del cual se entiende que la forma de convivencia dentro del hogar incida en el comportamiento fuera de él (Berger y Luckman, 2003).

\section{Estado de la cuestión y marco teórico}

Diversas investigaciones se han enfocado en analizar las relaciones violentas dentro de las familias (García y de Oliveira, 1994; Ramírez, 2007; Rojas, 2016; Serrano y Casique, 2013), teniendo como primera conclusión que la víctima principal suele ser la mujer, mientras quien ejerce la violencia es el hombre. En ese sentido, Berger y Luckman (2003) explican la socialización primaria como el aprendizaje de roles sociales, de normas y de valores dentro de las relaciones familiares, que son replicados en la socialización al exterior de estas.

En general, las investigaciones apuntan a que existe una asociación entre los ambientes de convivencia hostil en el hogar y los comportamientos violentos de los niños y jóvenes en otros espacios como la escuela y la vía pública, así como en sus relaciones de pareja cuando son mayores (Alvarado, 2014b; Concha-Eastman y Concha, 2014; Frías y Castro, 2011; Hoffman, Ireland y Widom, 1994; Ramírez, 2007). 
El estrato socioeconómico ha sido ampliamente abordado en términos de cómo influye en la existencia de ambientes violentos. Las investigaciones indican, en su mayoría, que el pertenecer a un estrato bajo incrementa la probabilidad de vivir en un entorno violento (Ramírez, 2007; Rojas, 2006), situación que se explica dentro del hogar como respuesta a una situación de tensión constante relacionada con la escasez de recursos.

De igual forma, en contextos como el mexicano, caracterizados por elevada violencia, donde gran parte de la población carece de recursos económicos básicos, empleos de calidad y oportunidades de desarrollo en general; involucrarse en actividades ilícitas, pero lucrativas, suele ser cada vez más frecuente. En particular, se ha demostrado que muchos de los jóvenes que se involucran en este tipo de situaciones también buscan satisfacer exigencias sociales de consumo (Concha-Eastman y Concha, 2014; Tourinho, Trassi, Decot y Tinoco, 2014).

Alvarado (2014b) encontró, tras analizar la situación en varias ciudades latinoamericanas, que en los barrios con mayor concentración de violencia tenían altos niveles de pobreza, precariedad laboral, desempleo, acceso a vivienda y a servicios básicos precarios. Además, esos mismos barrios tenían pocos procesos de participación ciudadana.

En cuanto a la importancia del entorno urbano, destaca el trabajo de Nava (2014), quien en una investigación realizada en Cancún, México, encontró que los jóvenes, independientemente de la forma de relacionarse en su familia, se sentían más seguros en casa que en cualquier otro lugar, debido a la situación imperante de violencia en las calles y en la escuela.

En la investigación social se ha acuñado el término "acumulación de desventajas" para referirse a factores de riesgo, materializados en situaciones o eventos negativos que llevan al individuo a transitar de forma errática por las etapas de su curso de vida, determinando trayectorias desafortunadas. La idea de "acumulación" deriva de que las desventajas se refuerzan mutuamente y pueden promover otras, generando un efecto conjunto de restricción de oportunidades y, en consecuencia, una aproximación a la exclusión social; en situaciones extremas, esta acumulación de desventajas podría implicar una ruptura de la relación individuo-sociedad. Desde esta perspectiva, los riesgos deben estudiarse en conjunto y no como situaciones aisladas, dando prioridad a las causas de los problemas para proponer medidas preventivas (Saraví, 2006a, 2006b, 2007, 2009).

Entre los distintos sectores sociales, los jóvenes son particularmente vulnerables a las desventajas, porque se encuentran en una etapa caracterizada por tener muchas transiciones, lo que multiplica la incertidumbre y la imprevisibilidad; entre esas transiciones está el cambio de una posición de subordinación por edad y dependencia económica a una de independencia. Aunado a lo anterior, las principales instituciones que permiten la movilidad e integración social, es decir, la familia, la escuela y el mercado de trabajo 
están debilitadas ${ }^{3}$, provocando que algunos jóvenes se enfrenten a estructuras de oportunidades fracturadas, débiles y discontinuas (Saraví, 2004, 2007) .

Debido a las condiciones heterogéneas de la sociedad, los jóvenes están expuestos a distintos riesgos y cuentan con herramientas diferentes para enfrentarlos, determinados por contextos particulares. Dentro de las familias y en las relaciones sociales en general, uno de los principales factores de riesgo de sufrir violencia es ocupar una posición subordinada, por edad, sexo o capacidades (Casique, 2012).

Entre las desventajas asociadas con situaciones de violencia destaca haber vivido violencia intrafamiliar, entendida como las agresiones físicas, psicológicas o sexuales ejercidas entre individuos con lazos de sangre o de afinidad, aunque ocurra en un espacio público (Frías y Castro, 2011; Morrison y Shifter, 2005). Según Corsi (1999), durante algunas décadas del siglo pasado dominó la idea de que lo acontecido en el interior de las familias debía quedar en la intimidad y correspondía al ámbito privado. Más adelante, estudios científicos sobre la violencia comenzaron a desarrollarse, enfocados sobre todo en el maltrato físico de los niños desde una perspectiva psicológica. Posteriormente, en la década de 1970, las feministas impulsaron la violencia de pareja como tema de discusión en instituciones internacionales, lo cual abrió la puerta a muchos estudios al respecto.

Berger y Luckman (2003) desarrollaron marcos teórico-conceptuales para explicar cómo los individuos asimilan su realidad social. Los procesos de asimilación y de inducción en la sociedad ocurren esencialmente en dos momentos: el primero, denominado socialización primaria, ocurre durante la infancia en el entorno familiar; el segundo, la socialización secundaria, acontece posteriormente y en otros espacios sociales.

Las formas de relacionarse, de entender el mundo, la autopercepción y el entendimiento de los otros, que son internalizados durante la socialización primaria, determinarán con más fuerza el actuar del individuo, debido a que durante muchos años será el único mundo conocido para él. Es un mundo dado y los niños no tienen suficientes elementos para cuestionarlo, de manera que suelen apropiarse de sus normas y significados casi automáticamente, porque la realidad aprendida en ese momento suele percibirse como inevitable (Berger y Luckman, 2003). Aplicado a los aprendizajes de conductas violentas, puede suponerse que si en una familia los conflictos se resuelven con agresiones o hay relaciones de subordinación entre los miembros, los niños y jóvenes internalizarán estas conductas como normales y eventualmente muchos las replicarán.

La socialización secundaria ocurre en etapas posteriores del desarrollo de los individuos, como la escuela y el trabajo, donde se internalizan realidades parciales y se adquiere un

3 El desempleo, la precarización e inestabilidad laborales, los nuevos arreglos e inestabilidad familiares y un incremento de la escolaridad que no logra reflejarse en el bienestar social son algunas de las condiciones actuales (Castel, 2004; Saraví, 2004). 
conocimiento específico de roles, vocabularios y significados, que habitualmente no son generalizables al "mundo de base" aprendido durante la socialización primaria. Incluso, la socialización secundaria presupone la existencia de una primaria en la cual se van a sobreponer los nuevos conocimientos (Berger y Luckman, 2003).

La desigualdad social en el contexto comunitario o la colonia puede incidir de manera significativa en la articulación de dichas desventajas, e incluso convertirse en una de ellas, en detrimento de quienes pertenecen a sectores populares.

La exclusión social, por otra parte, implica una completa marginación del sistema, provocada por la acumulación de muchas desventajas, en especial las relacionadas con la pobreza y la carencia de oportunidades. Lo que se observa con mayor frecuencia es un acceso parcial al sistema de algunos sectores sociales; estos sectores son excluidos solo de ciertos beneficios (como acceso a la salud, la educación, el empleo digno, entre otros), o su acceso es precario en comparación con otros grupos de la misma época y la misma sociedad, por lo se modifica la forma en la que experimentan la realidad (Paugam, 2001; Saraví, 2007).

Debido a la heterogeneidad social, en una misma ciudad pueden convivir dos tipos de jóvenes: por un lado, quienes gozan de ciertas ventajas como mayor nivel educativo, acceso a empleos de calidad y viven en colonias con suficientes ofertas recreativas; por otro, los jóvenes excluidos de los beneficios del desarrollo, que tienen baja escolaridad y se enfrentan a un mercado de trabajo precarizado, quienes a la vez tendrán más retos para enfrentar situaciones de violencia y menos oportunidades de integración social, es decir, algunos jóvenes pertenecientes a estratos bajos cuentan con menos herramientas para resolver conflictos pacíficamente y evitar la violencia (Alvarado, 2014b).

Es así como los contextos donde se conjunta la desigualdad social con otras desventajas incrementan el riesgo de que los jóvenes se vean involucrados en hechos violentos en varios ámbitos, lo cual genera un círculo vicioso de rezago y violencia. Ello se traduce en que, en el nivel del hogar, las relaciones intrafamiliares violentas se repliquen a través de las generaciones, convirtiéndose en desventajas para los integrantes; mientras en grupos con menos carencias ese tipo de relaciones no tenderán a perpetuarse con la misma intensidad (Tuirán, 2002).

En México, las condiciones de profunda desigualdad, junto con los procesos de exclusión, provocan que haya familias en zonas urbanas con características culturales similares a las indígenas o rurales; es decir que, a pesar de vivir en un contexto "modernizado", no gozan de todos los beneficios de este, como estabilidad económica y elevada escolaridad. Estas características son determinantes en las formas de organización familiar, y una de sus principales consecuencias es que las relaciones de pareja y con los hijos son menos democráticas entre individuos de sectores populares, quienes tienden a emplear la violencia como mecanismo de resolución de conflictos, lo cual incrementa los niveles de violencia intrafamiliar (Rojas, 2016). 
Por diversos motivos, las mujeres de todos los sectores se vinculan en el mercado laboral. Sin embargo, en sectores populares esto no garantiza la flexibilización de su rol doméstico ni incrementa su libertad para tomar decisiones. Principalmente en contextos conservadores y precarios, la incursión femenina en el empleo puede tener consecuencias como la generación de conflictos y de violencia en la pareja, pues los varones consideran amenazada su masculinidad por no poder cumplir su rol como proveedores económicos del hogar, o por estar en una posición inferior (educativa, laboral o económica), sea en relación con otros hombres o con su pareja. En consecuencia, la violencia masculina aparece como una forma de restaurar el orden de género, en el que el hombre tiene una superioridad jerárquica respecto a su esposa y a sus hijos (Rojas, 2016).

En contraste, entre familias de sectores medios, el empleo favorece la autonomía femenina, no incrementa el riesgo de sufrir violencia e incluso puede considerarse como una ventaja. Los varones con alta escolaridad de estos sectores suelen flexibilizar sus roles en la familia, participando más en las labores del hogar y cuidado de los hijos, aceptan que sus parejas son libres para tomar decisiones y realizar trabajos extradomésticos y en muchos de los casos las relaciones son cada vez más democráticas (Rojas, 2016).

De esta manera, la desigualdad social y el género se articulan y convierten en desventajas acumuladas, en especial para las mujeres de familias que tienen muchas carencias materiales. Por un lado, el empleo femenino es un factor de riesgo de conflictos entre las parejas pertenecientes a sectores populares, debido a que representa una amenaza para el orden de género establecido; en contraste, entre las familias de sectores medios el empleo conlleva el empoderamiento de las mujeres e incluso a la democratización de las relaciones entre los miembros del hogar (Rojas, 2016).

En un estudio elaborado a nivel internacional se pretendía analizar si las relaciones violentas en el interior del hogar están determinadas por roles y estereotipos de género, para lo cual se consideraron datos de noventa ciudades. Los resultados indican que las ciudades con mayores niveles de violencia intrafamiliar tenían normas familiares más rígidas que concedían una posición dominante a los hombres, y el uso de violencia física era socialmente aceptado. Es decir, la violencia en la familia no solo está enmarcada por los procesos dentro de ella, sino que también es determinada por la estructura social y cultural de género. Desafortunadamente, en el ámbito extradoméstico la realidad no es muy diferente, pues se sabe que las mujeres tienen mayor desventaja frente a los hombres en muchos aspectos, particularmente en cuanto al poder en las relaciones, pero también respecto a los niveles de ingreso y seguridad, los cuales suelen ser menores para ellas (Morrison y Shifter, 2005).

En América Latina existen importantes desigualdades en las expresiones de la violencia por género; Kessler y Dimarco (2014) dan cuenta de una transformación en las relaciones entre mujeres, las cuales se asemejan a las masculinas en términos de agresiones y 
peleas, aunque entre ellas los chismes son el principal motivo. En contraste, Tourinho et al. (2014) con la misma metodología destacan que los jóvenes varones brasileños afirman que las mujeres son responsables de las situaciones de violencia, e incluso que les gusta ser maltratadas, es decir, que justifican la violencia hacia ellas como una forma normal de relacionarse.

En el contexto mexicano, cuando se analizó a mujeres que han confrontado los roles o estereotipos de género, se concluyó que diversas formas de resistencia en vez de contrarrestar el problema podrían mantenerlo o incrementar los conflictos. Entre ellas puede señalarse el robo de dinero a la pareja, devolución de los golpes, emplear las relaciones sexuales como formas de chantaje e incluso las separaciones temporales (García y de Oliveira, 1994). En cambio, en otros estudios se han detectado cuestionamientos a las relaciones violentas mediante el diálogo con la pareja, para evitar peleas y agresiones entre algunas mujeres de sectores medios, urbanos y con alta escolaridad; incluso en casos donde los conflictos persisten y pueden llegar a una disolución del matrimonio (Rojas, 2016).

En un análisis elaborado a partir de la ENDIREH-2003, que pone énfasis en el género de los individuos relacionados con la violencia, se concluye que, en el ámbito escolar, los hombres suelen involucrarse en conflictos agresivos en mayor medida que las mujeres. El resultado indica que durante la niñez y la adolescencia los hombres son más vulnerables a ser violentados en sus principales ámbitos de socialización: la escuela y la familia (Frías y Castro, 2011).

En México aún se considera mayoritariamente vulnerables de ser víctimas a las mujeres, incluso como personas que viven doble o triple riesgo de ser agredidas por ser mujeres, jóvenes y pobres (Alvarado, 2014b). Sin embargo, es importante considerar los matices propios de cada región.

En ciudades como León, caracterizadas por tener poblaciones más conservadoras, las mujeres están convencidas de su mayor vulnerabilidad frente a los hombres, pese a que ellos son víctimas de robos en sus colonias. Muchas jóvenes no se atreven a salir de su casa si no las acompaña un varón, o solo recorren caminos conocidos y muy seguros, lo cual es un factor reductor de su autonomía (Vega, 2014).

El caso de Ciudad Juárez demuestra, por otra parte, que existen niveles de violencia en los que todos los jóvenes se vuelven vulnerables. De acuerdo con Alanís y Durán (2014), los jóvenes entrevistados de ambos sexos dijeron que, a pesar de que los medios de comunicación dan mayor difusión a los homicidios y desapariciones femeninos, los hombres también viven el riesgo de ser secuestrados o asesinados por pandillas o grupos del crimen organizado, lo cual alerta sobre la importancia que tiene la percepción subjetiva en los fenómenos de la violencia, así como los riesgos diferenciados por género. 
Por último, Alvarado (2014a) reporta que en la ciudad de México hay un miedo generalizado de viajar en transporte público y pasar por las zonas peligrosas de la ciudad, a ello las mujeres agregan el miedo permanente a las agresiones sexuales por su incapacidad para defenderse, lo cual es un indicador de que aunque todas las personas sean vulnerables a sufrir violencia, esta no se manifiesta en la misma forma para hombres y mujeres.

\section{Método}

A partir de la Encuesta de cohesión social para la prevención del delito (Ecopred) del 2014, se analizaron 32879 casos de jóvenes entrevistados a nivel nacional y 1316 en las alcaldías Iztapalapa y Gustavo A. Madero de la Ciudad de México. Se incluyó únicamente a los jóvenes que eran hijos del jefe del hogar y que respondieron a todas las preguntas empleadas para construir las variables incluidas en los modelos.

Se generaron variables para conocer las características de los jóvenes incluidos en el estudio en distintos niveles ${ }^{4}$. En el nivel individual se empleó una variable dicotómica para identificar el sexo de la persona (ser mujer es la categoría de referencia, una variable de la edad conformada por cuatro grupos: 12-14, 15-18, 19-22 y 23-29 [15-18 es la categoría de referencia] y ocupación, con cinco categorías: estudia, trabaja, estudia y trabaja, no estudia ni trabaja, solo quehaceres del hogar (estudiar es la categoría de referencia).

En el nivel del hogar se incluyeron seis variables, todas ellas de construcción propia. El tipo de hogar considera si es nuclear cuando en el hogar vive el jefe del hogar, su cónyuge y al menos un hijo; monoparental cuando solo vive el jefe del hogar con al menos un hijo; ampliado cuando vive el jefe del hogar, al menos un hijo y otra(s) persona(s) con o sin parentesco.

El estrato socioeconómico de los hogares se construyó a partir del método de componentes principales y de análisis de conglomerados. Con base en el nivel de hacinamiento del hogar (resultado de dividir el número de integrantes del hogar entre el número de cuartos destinados para dormir en la vivienda), el valor de escolaridad del jefe del hogar o su cónyuge (el que resultara más alto) y la posición en el trabajo del jefe o su cónyuge (el que tuvo la escolaridad más alta) se obtuvieron los componentes principales, siendo el primer componente el seleccionado para continuar con la construcción de la variable. Por medio de análisis de conglomerados con el método de k-medias se generaron tres medias en torno a las cuales se agruparon los valores para todos los hogares de los jóvenes incluidos en el estudio, con la finalidad de obtener tres grupos o estratos: muy

4 La generación de variables se basa en Pérez (2016), en este trabajo puede encontrarse una descripción más detallada del proceso. 
bajo, bajo y medio-alto, considerando que las características de la población mexicana nos indican que la probabilidad de que se incluyan hogares de estrato alto es muy baja.

Se generaron cuatro índices que permitirán caracterizar un ambiente de convivencia hostil o armonioso en el hogar. El índice de conflictividad del joven con otros miembros del hogar se basa en la frecuencia de conflictos que declaró haber tenido el joven con el resto de los integrantes del hogar (cuando adquiere valores altos indica ambiente de convivencia hostil, y con valores bajos indica ambiente armonioso). El índice de conflictividad entre los miembros del hogar se basa en la frecuencia de conflictos declarados por el joven donde él no participó (cuando adquiere valores altos indica convivencia hostil y viceversa). El índice de cohesión familiar se refiere a las formas de relacionarse, de resolver conflictos y si hay agresiones entre los miembros del hogar (cuando adquiere valores bajos indica convivencia hostil y viceversa). El índice de convivencia del joven con sus padres considera si realizan actividades juntos, si los jóvenes comentan sus problemas con sus padres, si estos ponen límites al comportamiento riesgoso de sus hijos, si están al pendiente de con quién salen y a dónde van (cuando adquiere valores bajos indica convivencia hostil y viceversa). Los cuatro índices fueron estandarizados para que tuvieran valores entre cero y diez, de manera que un ambiente de convivencia hostil extrema tendría: un índice de conflictividad con el joven con valor de diez, un índice de conflictividad sin el joven con valor de diez, un índice de cohesión familiar con valor de cero y un índice de convivencia con los padres con valor de cero.

Una vez identificada la presencia de conflictos, se asignaron valores a las frecuencias reportadas por el joven para cada situación, muy frecuente (10), frecuente (7.5), poco frecuente (5), casi nunca (2.5) y no declara (0). Una vez obtenidos los valores se sumaron los conflictos multiplicados por su frecuencia y se procedió a una estandarización:

$$
\text { Índice estandarizado }=\frac{\text { Valor observado }- \text { valor mínimo }}{\text { valor máximo }- \text { valor mínimo }} * 10
$$

A nivel del entorno se generó un índice de riesgo en la colonia, el cual refleja la percepción de los jóvenes sobre situaciones peligrosas, delictivas o violentas en el barrio o colonia donde habitan. Entre las situaciones se encuentran: ver personas bebiendo en la calle, gente vendiendo drogas, pandillerismo, robos y asaltos. El índice se estimó con el mismo proceso que los anteriores, y se estandarizó para que tuviera valores entre cero y diez, de manera que diez indica que los jóvenes observaron todos los riesgos posibles en su colonia, y cero que no observaron ninguno.

La variable explicada en el modelo estadístico es haber tenido conductas de riesgo alguna vez en su vida que pudieran relacionarse con actividades delictivas o criminales de las personas. Las conductas incluidas en esta variable son: 
- Haber tomado alguna bebida alcohólica hasta emborracharse para divertirse o porque le gusta.

- Haber fumado cigarros (tabaco) para experimentar, para tranquilizarse o por gusto.

- Haber consumido mota/hierba (marihuana) para experimentar, para tranquilizarse o por gusto.

- Haber inhalado thinner, PVC, cemento, resistol, pegamento, gasolina, solventes, chemos, etc., para experimentar, para divertirse o por gusto.

- Haber consumido algún tipo de alucinógeno como hongos, peyote, mezcalina, LSD, ácidos, etc., para experimentar, para divertirte o por gusto.

- Haber consumido éxtasis, tachas, cristal (anfetaminas), polvo, perico, nieve, grapa, cocaína, crack, piedra o heroína para experimentar, para divertirse o por gusto.

- Haber tenido alguna vez un arma para defenderse o para que lo respeten los demás.

- Haber pertenecido o haber sido obligado a pertenecer a algún grupo para proteger un territorio, para protegerse de otras personas o para que lo respeten.

- Haberse juntado con sus amigos para grafitear sin permiso una pared, romper vidrios, quemar algo, etc., por la emoción de hacerlo.

Con lo anterior, se construyeron dos modelos de regresión logística; uno para todas las ciudades consideradas en la encuesta y otro para las dos alcaldías de la Ciudad de México. Ambos modelos incluyen las diez variables explicativas en los tres niveles identificados.

\section{Resultados}

En la tabla 2 se presentan los resultados de los modelos logísticos a nivel nacional y a nivel Ciudad de México. Con excepción de la ocupación, todas las variables tuvieron la misma tendencia para ambos niveles de agregación.

A nivel individual, se comprobó que ser hombre incrementa los momios de haber tenido conductas de riesgo, la razón de momios es de 2.09 en comparación con las mujeres a nivel nacional y de 1.87 en las alcaldías de la Ciudad de México. Es decir, manteniendo el resto de las variables constantes, la probabilidad de que un joven varón haya tenido el tipo de conductas analizadas es menor en la Ciudad de México que en el país en su conjunto. Ser hombre representa una desventaja para los jóvenes, porque muchos de ellos, como resultado de los estereotipos de género, consideran tener conductas peligrosas o 
violentas como una forma de demostrar su virilidad, imponer su superioridad frente a los pares o ganar el respeto de las mujeres (Ramírez, 2005; Ramírez, 2007), sin embargo, el impacto de la variable resultó ligeramente menor para los varones capitalinos.

La edad resultó significativa en ambos modelos, con una tendencia creciente: a mayor edad, mayor riesgo de haber tenido conductas violentas, situación que se explica directamente por el mayor tiempo de exposición que han tenido los jóvenes de mayor edad. Sin embargo, se observa nuevamente que en la Ciudad de México la diferencia entre las categorías es menor que la observada a nivel nacional.

La ocupación es la variable con mayor diferencia entre ambos modelos. En el modelo aplicado a las alcaldías de la Ciudad de México, las distintas ocupaciones no resultaron ser estadísticamente distintas respecto a ser estudiante (variable de referencia). A nivel nacional, todas las categorías conllevan mayores momios para haber tenido conductas de riesgo en comparación con estudiar; con excepción de quienes no estudian ni trabajan, todas las categorías fueron estadísticamente significativas. Esta variable nos indica que la exposición al riesgo en relación con la ocupación no resulta menos relevante en el nivel local en comparación con el nivel nacional.

En el nivel nacional, por lo tanto, trabajar es una desventaja para los jóvenes, mientras estudiar es una ventaja que les permite mantenerse alejados o afrontar situaciones adversas (Alvarado, 2014a), entre las que se encuentran las propiciadoras de que los jóvenes tengan conductas de riesgo. $\mathrm{Al}$ considerar el tiempo de exposición al riesgo debemos recordar que quienes trabajan o estudian y trabajan y pertenecen, en su mayoría, al grupo de mayor edad, presentan de manera acumulada ambas desventajas: la edad y la ocupación.

En el nivel del hogar, los jóvenes que viven en familias con estructuras distintas a la monoparental tienen más momios de haber tenido conductas de riesgo en ambos modelos, lo que coincide con investigaciones previas, donde se considera que las familias monoparentales son un factor de riesgo para los jóvenes (Frías y Castro, 2011; Rodríguez, 2001). Cabe destacar que en la Ciudad de México esta variable, pese a presentar la misma tendencia que la nacional, no demostró tener diferencias estadísticamente significativas entre sus categorías.

En cuanto al estrato socioeconómico, los resultados indican que los jóvenes de hogares pertenecientes al bajo poseen el menor riesgo de haber tenido este tipo de conductas $(17 \%$ menos que los de estrato medio-alto a nivel nacional y $19 \%$ menos a nivel local), y los jóvenes del estrato muy bajo tienen el efecto opuesto ( $21 \%$ más que los de estrato medio-alto a nivel nacional y $1 \%$ a nivel local). Es decir, pertenecer a un estrato socioeconómico muy bajo es una desventaja para los jóvenes, en contraste, pertenecer al bajo es una ventaja. Esta variable tampoco resultó ser estadísticamente significativa para los jóvenes de Iztapalapa y Gustavo A. Madero. 
Factores que incrementan el riesgo de que los jóvenes se vean involucrados en situaciones violentas o delictivas ...

Tabla 2. Modelo logístico para haber tenido conductas de riesgo

\begin{tabular}{|c|c|c|c|c|c|c|}
\hline \multirow[t]{2}{*}{ Variable } & \multicolumn{3}{|c|}{ Nacional } & \multicolumn{3}{|c|}{ CDMX } \\
\hline & $\begin{array}{l}\text { Razón de } \\
\text { momios }\end{array}$ & \multicolumn{2}{|c|}{ Intervalo de confianza } & $\begin{array}{l}\text { Razón de } \\
\text { momios }\end{array}$ & \multicolumn{2}{|c|}{ Intervalo de confianza } \\
\hline Constante & $2.18 * * *$ & 1.79 & 2.66 & $6.68 * * *$ & 2.49 & 17.92 \\
\hline \multicolumn{7}{|l|}{ Sexo } \\
\hline Mujer & \multicolumn{3}{|c|}{ Referencia } & \multicolumn{3}{|c|}{ Referencia } \\
\hline Hombre & $2.09 * * *$ & 1.98 & 2.20 & $1.87 * * *$ & 1.44 & 2.43 \\
\hline \multicolumn{7}{|l|}{ Grupo de edad } \\
\hline $12-14$ & $0.33^{* * *}$ & 0.30 & 0.35 & $0.30 * * *$ & 0.19 & 0.45 \\
\hline $15-18$ & \multicolumn{3}{|c|}{ Referencia } & \multicolumn{3}{|c|}{ Referencia } \\
\hline $19-22$ & $2.11 * * *$ & 1.97 & 2.27 & $1.92^{* * * *}$ & 1.37 & 2.69 \\
\hline $23-29$ & $2.38 * * *$ & 2.20 & 2.58 & $2.08 * * *$ & 1.42 & 3.04 \\
\hline \multicolumn{7}{|l|}{ Ocupación } \\
\hline Estudia & \multicolumn{3}{|c|}{ Referencia } & \multicolumn{3}{|c|}{ Referencia } \\
\hline Trabaja & $1.27 * * *$ & 1.18 & 1.37 & 1.07 & 0.76 & 1.50 \\
\hline Estudia y trabaja & $1.17 *$ & 1.03 & 1.32 & 0.95 & 0.51 & 1.77 \\
\hline Ni estudia, ni trabaja & 1.02 & 0.87 & 1.20 & 0.71 & 0.35 & 1.44 \\
\hline $\begin{array}{l}\text { Solo quehaceres del } \\
\text { hogar }\end{array}$ & $1.17 * *$ & 1.05 & 1.30 & 1.11 & 0.71 & 1.72 \\
\hline \multicolumn{7}{|l|}{ Tipo de hogar } \\
\hline Nuclear & \multicolumn{3}{|c|}{ Referencia } & \multicolumn{3}{|c|}{ Referencia } \\
\hline Monoparental & $1.22 * * *$ & 1.14 & 1.31 & 1.22 & 0.88 & 1.70 \\
\hline Ampliado & $1.19 * * *$ & 1.11 & 1.27 & 1.26 & 0.92 & 1.73 \\
\hline \multicolumn{7}{|l|}{ Estrato socioeconómico } \\
\hline Muy bajo & $1.21 * * *$ & 1.14 & 1.28 & 1.01 & 0.76 & 1.35 \\
\hline Bajo & $0.83 * * *$ & 0.77 & 0.90 & 0.81 & 0.59 & 1.13 \\
\hline Medio-alto & \multicolumn{3}{|c|}{ Referencia } & \multicolumn{3}{|c|}{ Referencia } \\
\hline \multicolumn{7}{|c|}{ Índices de convivencia en el hogar } \\
\hline $\begin{array}{l}\text { Conflictividad con otros } \\
\text { miembros del hogar }\end{array}$ & $1.23^{* * *}$ & 1.18 & 1.29 & $1.25 *$ & 1.02 & 1.53 \\
\hline $\begin{array}{l}\text { Conflictividad entre } \\
\text { otros miembros }\end{array}$ & $1.20 * * *$ & 1.15 & 1.25 & $1.34^{* *}$ & 1.09 & 1.65 \\
\hline Cohesión familiar & $0.81 * * *$ & 0.79 & 0.82 & $0.80 * * *$ & 0.74 & 0.87 \\
\hline $\begin{array}{l}\text { Convivencia del joven } \\
\text { con sus padres }\end{array}$ & $0.91^{* * *}$ & 0.89 & 0.92 & $0.83^{* * *}$ & 0.75 & 0.91 \\
\hline \multicolumn{7}{|l|}{ Índice de la colonia } \\
\hline $\begin{array}{l}\text { Percepción de riesgo en } \\
\text { la colonia }\end{array}$ & $1.11 * * *$ & 1.10 & 1.12 & $1.10^{* * *}$ & 1.05 & 1.15 \\
\hline
\end{tabular}

Nota: * $\mathrm{p}<0.05 ; * * \mathrm{p}<0.01 ; * * * \mathrm{p}<0.001$

Fuente. Elaboración propia con base en la Ecopred-2014 
Los cuatro índices asociados a ambientes de hostilidad en el hogar resultaron estadísticamente significativos y demuestran tener el impacto esperado en la conducta de los jóvenes. Los dos índices de conflictividad tienen una correlación positiva con la probabilidad de que los jóvenes hayan tenido alguna conducta de riesgo, lo cual indica que mientras más elevada sea la conflictividad en el hogar de un joven. este tendrá mayor propensión a involucrarse en situaciones de violencia. Estos resultados son congruentes con las afirmaciones de Frías y Castro (2011), quienes concluyen que los jóvenes tienen más riesgo de ser agresores si son violentados en casa. En el nivel nacional el impacto de los dos índices es muy similar (con razones de momios de 1.23 y 1.20); en la Ciudad de México tuvo mayor impacto la conflictividad entre otros miembros del hogar (1.34) que la conflictividad donde el joven es partícipe (1.25).

El índice de cohesión social y el índice de convivencia con los padres, por otra parte, tienen una correlación negativa con las conductas de riesgo. Por cada incremento en el valor del índice de cohesión familiar, la probabilidad de que el joven haya tenido conductas de riesgo disminuye $19 \%$ en el nivel nacional y $20 \%$ en el nivel local, mientras el índice de convivencia con los padres la reducirá en $9 \%$ en el nivel nacional y $17 \%$ en el nivel local. Ambos indican que cuando los jóvenes viven en ambientes más armoniosos hay una menor propensión a que tengan conductas riesgosas, por lo que ambos representan una ventaja para los jóvenes, tanto en el ámbito nacional como local.

Figura 1. Probabilidad de que los jóvenes varones hayan tenido conductas de riesgo según índices de conflictividad en el hogar

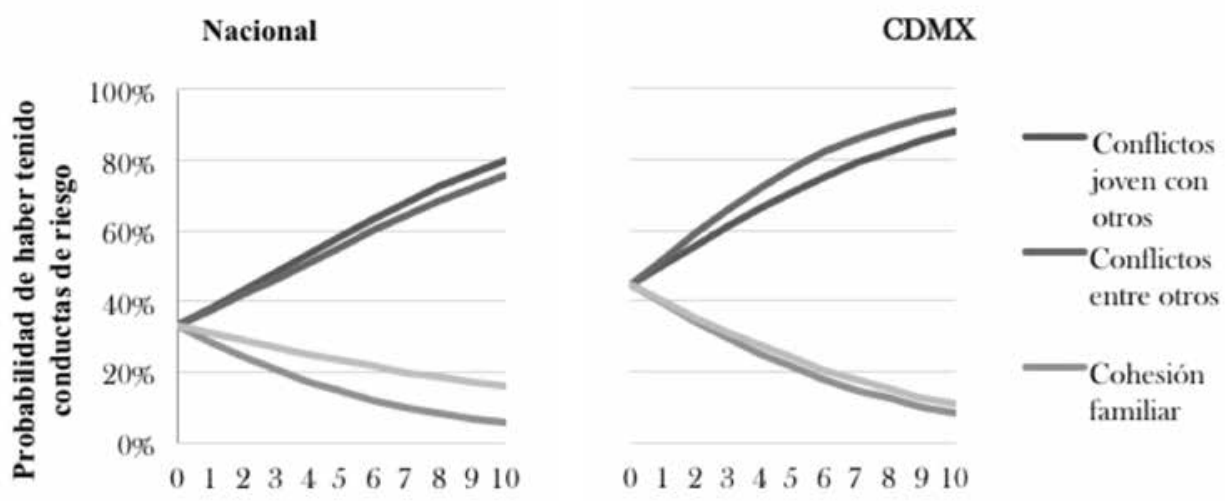

Fuente. Elaboración propia a partir de la tabla 2

En la figura se presentan las probabilidades de que los jóvenes hayan tenido conductas de riesgo según el valor de los índices de conflictividad en el hogar, considerando a los varones de estrato medio-alto, estudiantes, de entre 15 y 18 años, manteniendo el resto 
de las variables constantes. Destaca que en la Ciudad de México el impacto de las relaciones conflictivas entre otros miembros del hogar (conflictos entre otros) tiene un impacto tal que, si se presenta la mayor frecuencia posible, la probabilidad de que un joven con las características mencionadas tenga conductas de riesgo es del $94 \%$ frente al $76 \%$ a nivel nacional. La cohesión familiar resulta ser el índice más favorable para evitar que un joven tenga conductas de riesgo, tanto a nivel nacional como local, convirtiéndolo en la ventaja más importante en el nivel del hogar.

Por último, en el nivel de análisis del entorno, el índice de percepción de riesgos en la colonia tiene un impacto positivo y significativo en ambos modelos. Esto indica que, entre los jóvenes, tanto en el país como en la Ciudad de México, la percepción de altos riesgos en la colonia es una desventaja asociada a un mayor involucramiento en situaciones de violencia.

\section{Conclusiones}

Los resultados indican que algunas variables pueden ser consideradas definitivamente como desventajas: ser hombre, pertenecer a los grupos de mayor edad, tener una ocupación distinta a estudiar, pertenecer a un hogar monoparental o ampliado, pertenecer a un hogar de estrato muy bajo o medio-alto, vivir en un ambiente hostil de convivencia en el hogar (elevada conflictividad, participe el joven o no), vivir en una colonia que se perciba como riesgosa.

En contraste, las ventajas identificadas son: pertenecer a uno de los grupos de menor edad, tener como ocupación únicamente estudiar, pertenecer a un hogar de estrato socioeconómico bajo, vivir en un hogar con alta cohesión familiar y donde el joven tenga buena convivencia con sus padres.

En el nivel individual en ambos modelos se encontró que ser hombre es una de las desventajas significativas; por lo tanto, se comprueba que las relaciones de género siguen siendo determinantes en las conductas de los individuos. En respuesta a esta situación se recomienda que las políticas públicas y programas sociales orientados a prevenir conductas delictivas o violentas tengan una perspectiva de género, que considere que las decisiones de los jóvenes, en particular de los varones, responden a la presión social por cumplir estereotipos acordes a su género.

Además, deben aplicarse a edades tempranas, cuando los jóvenes han estado expuestos menos tiempo a la socialización primaria violenta y a otros riesgos. De acuerdo con Palacios (2008), es normal que la frecuencia, severidad y variedad de conductas de riesgo aumenten con la edad, se puede esperar que cuando los jóvenes han salido de la adolescencia empiecen a reducir su participación en este tipo de comportamientos. 
En términos académicos queda pendiente un análisis longitudinal que se enfoque en identificar conductas de riesgo entre los jóvenes de mayor edad, con el objetivo de identificar jóvenes que persisten con conductas de riesgo durante su vida, y si dichas conductas derivaron en la comisión de delitos.

A nivel nacional, trabajar fue la ocupación que implicaba más riesgo, en contraste con aquellos jóvenes que no tienen trabajo ni empleo, de manera que debe considerarse una prioridad no estigmatizar a las personas por no pertenecer al sistema educativo o laboral cuando se aborde el tema de las conductas violentas.

En cuanto a las ventajas y desventajas identificadas en el hogar, el estrato socioeconómico tiene una incidencia significativa a nivel nacional, pero no significativa en las alcaldías de la Ciudad de México analizadas. Los jóvenes de estrato muy bajo son los que tienen mayor propensión a involucrarse en situaciones de violencia, seguidos de los de estrato medio-alto y los menos involucrados son los de estrato bajo. Así, el pertenecer a los estratos extremos de la estratificación (muy bajo o medio-alto) provoca que los jóvenes sean más vulnerables a tener conductas de riesgo.

Los índices de convivencia resultaron tener el impacto más significativo en ambos modelos. Los hallazgos confirman que vivir en un hogar con un ambiente de convivencia hostil incrementa la probabilidad de que los jóvenes hayan tenido conductas de riesgo, mientras que vivir en un hogar armonioso reduce dicha probabilidad.

Un ambiente hostil de convivencia en el hogar favorece la réplica de conductas violentas en otros espacios de socialización, pues la socialización primaria se replicará en otros ámbitos de la vida de los individuos. En los modelos nacional y local los dos índices de conflictividad entre los miembros del hogar demostraron aumentar la probabilidad de que el joven se viera involucrado en situaciones de riesgo; mientras que el índice de cohesión familiar y el de convivencia con los padres denotan que un ambiente armonioso reduce la misma probabilidad.

Por lo anterior, la correlación de la presencia de conflictos entre los miembros del hogar y la ausencia de cohesión familiar con mayor probabilidad de tener conductas de riesgo permite afirmar que entre las consecuencias de que la socialización primaria ocurra en un ambiente hostil para los jóvenes, se encuentra el posterior involucramiento en situaciones de riesgo, de violencia o delictivas.

La razón por la que valores altos del índice de convivencia con los padres se asocian con la victimización de los jóvenes no puede ser explicada por la teoría sociológica de dichos autores, sin embargo, el tema ha sido abordado desde la psicología social por investigadores como Andrade y Betancourt (2008), quienes vinculan la sobreprotección de los niños y jóvenes con mayor vulnerabilidad. Para poder mejorar el índice de convivencia con los padres podrían incluirse factores sobre cómo se sienten los jóvenes con el tipo de 
relación que tienen con sus padres, de tal manera que se identifique si la cercanía con ellos funge como factor protector o disminuye las facultades de los jóvenes para defenderse de agresiones en el ámbito social.

En el nivel de análisis de la colonia el índice de percepción de riesgo también tuvo el resultado esperado: vivir en colonias de alta peligrosidad es una desventaja para los jóvenes. Es posible afirmar que incluir desventajas que pudieran afectar a los jóvenes en los tres niveles de análisis fue adecuado. Por lo tanto, se recomienda incluir en otras investigaciones características del entorno social, o de la colonia, en las investigaciones sobre conductas violentas, no únicamente para incrementar el horizonte explicativo de los modelos estadísticos, sino porque a ese nivel es posible generar políticas públicas de mayor alcance que reduzcan y prevengan los actos delictivos o violentos.

Cabe destacar que se identificaron menos desventajas para los jóvenes de la Ciudad de México que pudieran explicar las conductas de riesgo; características como la ocupación, el tipo de hogar y el estrato socioeconómico no tuvieron significancia estadística pese a que la tuvieron a nivel nacional.

A lo largo de la investigación se pudo constatar la complejidad de las conductas y relaciones hostiles entre las personas. Se reconoce que políticas públicas aisladas no servirán para combatir la forma en que la violencia se replica entre individuos y a través de los espacios de socialización. Es necesario, más que combatir las consecuencias que se encuentran al final de la cadena de acumulación de desventajas, combatir las desventajas identificadas que afectan a los jóvenes, considerando las interacciones entre ellas (Saraví, 2006b).

En el mismo sentido, se requiere considerar las características y las ventajas con las que cuentan los jóvenes, que pueden ayudarlos a enfrentar sus problemas de manera pacífica. Entre otras acciones se sugiere: estimular una convivencia armoniosa en hogares ubicados en ciudades conflictivas, generar programas focalizados a la solución de conflictos en escuelas primarias y secundarias, realizar acciones barriales en las que la percepción de los habitantes sobre su misma colonia mejore.

\section{Referencias bibliográficas}

Alanís, Ú. y Durán, A. (2014). Jóvenes en Ciudad Juárez, Chihuahua: entre la falta de oportunidades y el miedo a la violencia. En A. Alvarado (ed.), Violencia fuvenil y Acceso a la fusticia en América Latina (pp. 63-112). México: El Colegio de México.

Alvarado, A. (2014a). Violencia Fuvenil y Acceso a la Justicia en América Latina. México: El Colegio de México. 
Revista CIFE / ISSN: 0124-3551 e-ISSN: 2248-4914 / Bogotá-Colombia / Vol. 21 N.o 34 / enero-junio 2018 / pp. $93-114$

Alvarado, A. (2014b). Los jóvenes, la violencia y la interacción con la policía en la ciudad de México. Violencia Juvenil y Acceso a la Justicia en América Latina. México: El Colegio de México.

Andrade, P. y Betancourt, D. (2008). Factores individuales, familiares y sociales y conductas de riesgo en adolescentes. Investigaciones psicosociales en adolescentes. México: UNICACH, UNAM.

Berger, P. y Luckman, T. (2003). La sociedad como realidad subjetiva. En P. Berger y T. Luckman (eds.), La construcción de la realidad social (pp. 162-225). Buenos Aires: Amorrortu Editores.

Casique, I. (2012). Vulnerabilidad a la violencia doméstica. Una propuesta de indicadores para su medición. Realidad, datos y espacio, Revista Internacional de Estadística y Geografia, 3(2), 46-65.

Concha, A. y Concha, N. (2014). Entre la desesperanza y la supervivencia. Realidades de los adolescentes y jóvenes de barriadas populares en Cali, Colombia, 2011. En A. Alvarado (ed.), Violencia Juvenil y Acceso a la Justicia en América Latina (pp. 215-318). México: El Colegio de México.

Corsi, J. (1999). Una mirada abarcativa sobre el problema de la violencia familiar. En J. Corsi (ed.), Violencia familiar. Una mirada interdisciplinaria sobre un grave problema social (pp. 15-63). Buenos Aires: Paidós.

ENVIPE (2018). Encuesta Nacional de Victimización y Percepción sobre Seguridad Pública 2018. INEGI. Recuperado de https://www.inegi.org.mx/programas/envipe/2018/

Frías, S. y Castro, R. (2011). Socialización y violencia: desarrollo de un modelo de extensión de la violencia interpersonal a lo largo de la vida. Estudios Sociológicos, 29(86), 497-550.

García, B. y de Oliveira, O. (1994). Jefas de hogar y violencia doméstica. En B. García y O. de Oliveira (eds.), Trabajo femenino y vida familiar en México (pp. 151-170). México: El Colegio de México.

Hoffman, J. P., Ireland, T. O. y Widom, C. S. (1994). Traditional Socialization Theories of Violence: A Critical Examination. En J. Archer (ed.), Male Violence (pp. 289-309). Londres: Routledge.

INEGI (2014). Encuesta de Cohesión Social para la Prevención de la Violencia y la Delincuencia (ECOPRED) 2014. Instituto Nacional de Estadística y Geografía. Recuperado de https://www.inegi.org.mx/programas/ecopred/2014/default.html 
Kessler, G. y Dimarco, S. (2014). Los jóvenes, la violencia y la interacción con la policía en Buenos Aires. En A. Alvarado (ed.), Violencia fuvenil y Acceso a la fusticia en América Latina (pp. 41-134). México: El Colegio de México.

Morrison, M. y Shifter, M. B. (2005). América violenta: factores de riesgo, consecuencias e implicaciones para las políticas sobre violencia social y doméstica. En H. Frühling, J. Tulchin y H. Golding (eds.), Crimen y violencia en América Latina (pp. 117-151). Bogotá: Fondo de Cultura Económica.

Nava, A. (2014). Los jóvenes, la violencia y la policía: el caso de Cancún. En A. Alvarado (ed.), Violencia Fuvenil y Acceso a la fusticia en América Latina (pp. 21-62). México: El Colegio de México.

Palacios, J. R. (2008). Covariación y desarrollo de múltiples conductas problema en adolescentes. En J. R. Palacios (ed.), Investigaciones psicosociales en adolescentes (pp. 147-179). México: UNICACH, UNAM.

Paugam, S. (2001). Les formes contemporaines de la pauvreté et de l'exclusion en Europe. Études rurales, 159/160, 73-95.

Pérez, A. (2016). Desventajas acumuladas e involucramiento juvenil en situaciones de violencia en ciudades de alta delictividad en México, 2014. (Tesis de maestría). El Colegio de México, Ciudad de México, México.

Ramírez, J. C. (2005). Madejas entreveradas: violencia, masculinidad y poder. Varones que ejercen violencia contra sus parejas. Guadalajara: Universidad de Guadalajara, Plaza y Valdés.

Ramírez, M. A. (2007). Hombres violentos. Un estudio antropológico de la violencia masculina. México: Plaza y Valdés.

Rodríguez, J. (2001). Vulnerabilidad y grupos vulnerables: un marco de referencia conceptual mirando a los jóvenes. Población y Desarrollo, 17. Santiago de Chile: CELADE-CEPAL.

Rojas, O. (2016). Mujeres, hombres y vida familiar en México. Persistencia de la inequidad de género anclada en la desigualdad social. Estudios de género, 3, 73-101.

Saraví, G. A. (2004). Juventud y violencia en América Latina. Reflexiones sobre exclusión social y crisis urbana. Desacatos, (14), 127-142.

Saraví, G. A. (2006a). Atmósfera familiar y transición a la adultez. Factores de riesgo asociados con transiciones tempranas. En R. Esteinou (ed.), Fortalezas y desafios de las familias en dos contextos: Estados Unidos de América y México (pp. 341-383). México: CIESAS. 


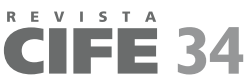

Revista CIFE / ISSN: 0124-3551 e-ISSN: 2248-4914 / Bogotá-Colombia / Vol. 21 N.o 34 / enero-junio 2018 / pp. $93-114$

Saraví, G. A. (2006b). Los eslabones de la violencia juvenil: acumulación de desventajas en la transición a la adultez. En J. Moro (ed.), Fuventudes, violencia y exclusión: desafios para las políticas públicas (pp. 89-129). Guatemala: Magna Tierra editores.

Saraví, G. A. (2007). Nuevas realidades y nuevos enfoques: exclusión social en América Latina. En G. Saraví (ed.), De la pobreza a la exclusión: continuidades y rupturas de la cuestión social en América Latina (pp. 19-52). Buenos Aires: Prometeo Libros.

Saraví, G. A. (2009). Transiciones vulnerables: juventud, desigualdad y exclusión en México. México: CIESAS.

Secretariado Ejecutivo del Sistema Nacional de Seguridad Pública (2019). Incidencia delictiva municipal nueva metodología, Secretaría de Gobernación. Recuperado de https:/ / datos.gob.mx/busca/dataset/secretariado-ejecutivo-del-sistema-nacional-de-seguridad-publica/resource/741dd72b-5487-49f5-86fl-aa3c1f87b4a9

Serrano, O. y Casique, I. (2013). Caracterización sociodemográfica de la muestra de la ENDIREH 2011 y comparación con la ENDIREH 2006 y 2003. En I. Casique y R. Castro (eds.), Expresiones y contextos de violencia contra las mujeres en México. Análisis comparativo de la ENDIREH 2011 (pp. 58-97). México: CRIM-INMUJERES.

Tourinho, F., Trassi, M. de L., Decot, M. y Tinoco, R. (2014). Violencia, adolescencia y juventud en Sao Paulo. En A. Alvarado (ed.), Violencia Juvenily Acceso a la fusticia en América Latina (pp. 137-211). México: El Colegio de México.

Tuirán, R. (2002). Transición demográfica, trayectorias de vida y desigualdad social en México: lecciones y opciones. Papeles de población, 8(31), 25-66.

Vega, J. (2014). La ciudad y la violencia que experimentan los jóvenes. El caso de León, Guanajuato. En A. Alvarado (ed.), Violencia fuvenil y Acceso a la fusticia en América Latina (pp. 113-164). México: El Colegio de México. 